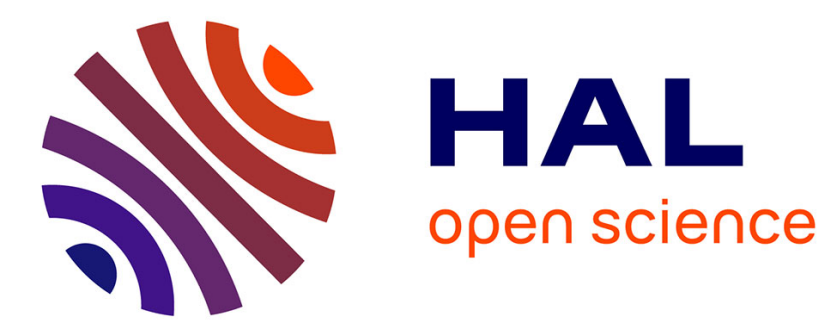

\title{
Interfacial properties of amphiphilic systems
}

\author{
M. Schick
}

\section{To cite this version:}

M. Schick. Interfacial properties of amphiphilic systems. Journal de Physique IV Proceedings, 1993, 03 (C1), pp.C1-47-C1-56. 10.1051/jp4:1993105 . jpa-00251548

\section{HAL Id: jpa-00251548 https://hal.science/jpa-00251548}

Submitted on 1 Jan 1993

HAL is a multi-disciplinary open access archive for the deposit and dissemination of scientific research documents, whether they are published or not. The documents may come from teaching and research institutions in France or abroad, or from public or private research centers.
L'archive ouverte pluridisciplinaire HAL, est destinée au dépôt et à la diffusion de documents scientifiques de niveau recherche, publiés ou non, émanant des établissements d'enseignement et de recherche français ou étrangers, des laboratoires publics ou privés. 


\title{
Interfacial properties of amphiphilic systems
}

\author{
M. SCHICK
}

Department of Physics FM-15, University of Washington, Seattle Washington 98195, U.S.A.

The standard Landau theory of interfacial properties is reviewed and seen to be inadequate to describe the wetting properties of amphiphilic systems. A simple extension of it is introduced and shown to provide a good description of recent experimental results. The origin of ideal middle phase behavior is shown to be a complete unbinding transition of surfactant sheets. This middle phase is an excellent solubilizer, and produces a zero interfacial tension between coexisting oil and water phases.

I shall discuss ternary mixtures of oil, water, and amphiphile, and how the observed behaviors of the interfaces between the various phases are a direct consequence of the nature of the amphiphile itself. For this purposes, an amphiphile can be taken to be a molecule which, by construction, has a part which likes water and another part which likes oil. Hence "amphiphile", from the Greek meaning loving both. These molecules try to make interfaces between the oil and water at which they can sit and lower their energy. In such ternary mixtures, one oberves several phases of which three liquid ones, which can all coexist, will be of interest to us here; a water-rich phase, an oil-rich phase, and a "middle" phase, which contains both water and oil, and more amphiphile than the other phases. An amphiphile is considered to be a good one if the concentration of amphiphile in this middle phase is rather small, for then only a small amount of it is needed to solubilize the other two components. Similarly, if the concentration of amphiphile in the middle phase is rather large, the amphiphile is considered to be a poor one.

What are the behaviors to be explained? Consider, first, a balanced system, one in which the concentration of amphiphile in the oil-rich and water-rich phases is the same. If the amphiphile is a good one, then the middle phase does not wet the interface between the oil- and water-rich phases. In a first experiment (1), one substitutes poorer and poorer amphiphiles, and finds that the contact angle of the pendant drop of middle phase decreases monotonically to zero, at which point the middle phase does wet the interface. For a second experiment, let us stay with the system of a good amphiphile and change the temperature so that the system becomes unbalanced. The compositions in the middle phase will approach those of one of the other phases, and these two will eventually become critical in the presence of the third at a critical endpoint. As John Cahn pointed out long ago (2), one expects a wetting transition to occur before the endpoint is reached. The results of the experiment (3) are shown in Fig. 1 where 
one sees that the behavior of the contact angle is certainly not monotonic and, for two of the amphiphiles. no wetting is observed as one or both of the endpoints is approached. In light of the Cahn argument, this is somewhat disconcerting.

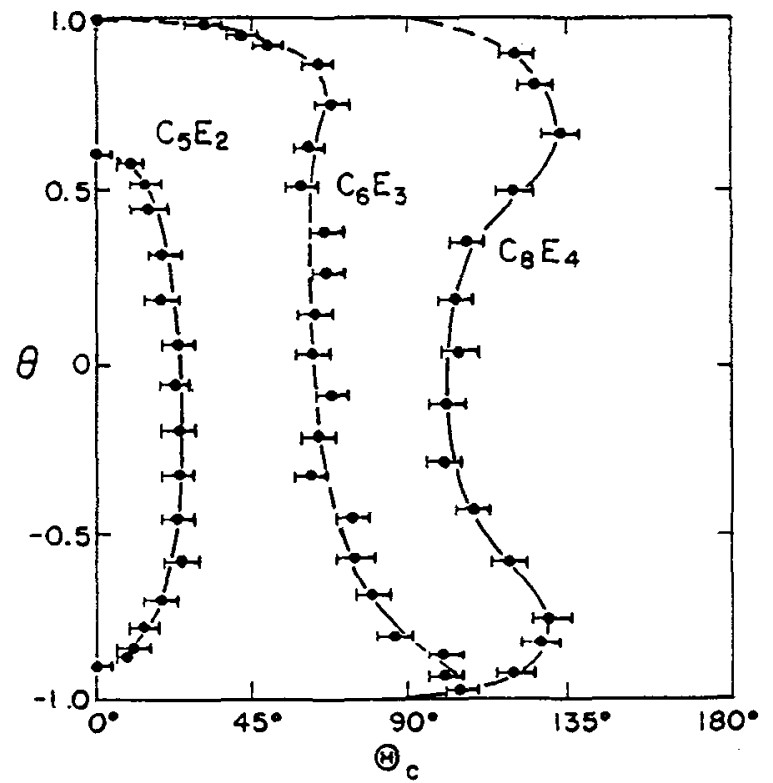

Fig. 1 Behavior of the contact angle $\Theta_{c}$ vs. reduced temperature $\theta$ for three different systems. The critical endpoints are at $\theta= \pm 1$. After Ref. 3.

In order to understand this behavior, the usual Landau free energy functional will be employed. First, let us recall how it works in normal fluid systems. The fluid is described by a one-component order parameter $M(\mathbf{r})$ which we can think of as the local concentration difference between oil and water. The Landau free energy functional of this order parameter is

$$
F\{M(\mathbf{r})\}=\int d^{3} r\left[g(\nabla M)^{2}+f(M)\right]
$$

The first term of the above ensures that spatial variations of the order parameter are costly, so that uniform bulk phases are preferred by this free energy. Information about the bulk phases are contained in $f(M)$, the bulk free energy per unit volume. If there were one bulk phase characterized by the value $M_{0}$ everywhere, then for $M$ close to $M_{0}$

$$
f(M) \approx \omega\left(M-M_{0}\right)^{2}
$$

The absolute minimum of Eq. 1 with this $f(M)$ is attained when $M=M_{0}$ everywhere. Fluctuations about this value are easily calculated. If the local value of $M$ is expanded about its average value

$$
M(\mathbf{r})=M_{0}+\sum_{\mathbf{q}} M_{\mathbf{q}} \exp (i \mathbf{q} \cdot \mathbf{r})
$$

then one finds that the ensemble average of $M_{\mathrm{q}} M_{-\mathbf{q}}$ which is proportional to the structure factor $S(\mathbf{q})$ is a Lorentzian

$$
<M_{\mathrm{q}} M_{-\mathrm{q}}>\propto \frac{1}{g q^{2}+w}
$$


and its Fourier transform, the correlation function, decays exponentially

$$
G(\mathbf{r})=\frac{2 \pi^{2}}{g r} \exp (-r / \xi),
$$

where the correlation length

$$
\xi=\sqrt{(g / \omega)}
$$

So much for one phase.

Let us now suppose that we have two phases in coexistence, one characterized by $M=M_{0}$, the other by $M=-M_{0}$. This situation can be described by

$$
f(M)=\left(M-M_{0}\right)^{2}\left(M+M_{0}\right)^{2} .
$$

Let the two phases be in coexistence, with an interface in the $x y$ plane. The order parameter profile will now vary with the $z$ coordinate in such a way that $M(z)$ will approach $\pm M_{0}$ as $z$ approaches $t \infty$. The desired profile is the function $M(z)$ which minimizes Eq. (1), or equivalently,

$$
\sigma\{M\} \equiv \int d z\left[g\left(\frac{d M}{d z}\right)^{2}+f(M)\right]
$$

subject to the given boundary conditions. One gains intuition by noting that this problem is, mutatis mutandi, equivalent to minimizing the action of a particle of mass $m$ moving in a potential $V$,

$$
S \equiv \int d t\left[\frac{m}{2}\left(\frac{d x}{d t}\right)^{2}-V(x)\right]
$$

Note in particular that $f(M)$ plays the role of $-V(x)$ so that our original problem with two minima is analogous to a classical particle in a region with two hills. Our boundary conditions are equivalent to requiring that the particle start from rest on one hill and, after being given an infinitesimal shove, come to rest on the other hill. The trajectory $x(t)$ is identical to the profile $M(z)$.

The Euler Lagrange equation which minimizes the action (9) is, of course, Newton's law

$$
m \frac{d^{2} x}{d t^{2}}=-\frac{d V}{d x}
$$

with the first integral

$$
\frac{m}{2}\left(\frac{d x}{d t}\right)^{2}+V(x)=E
$$

In our case, with the potential being the negative of $f(M)$ of Eq. (7), the energy of the particle is zero, and Eq. (11) leads to

$$
t=\int_{0}^{x}\left[\frac{m}{-2 V(x)}\right]^{1 / 2} d x
$$

which can be inverted to obtain the trajectory $x(t)$. When this is carried out for the explicit form of the potential obtained from Eq. (7) and reexpressed in terms of the variables of the original statistical mechanics problem, one obtains the familiar profile

$$
M(z)=M_{0} \tanh (z / \xi)
$$

With this profile, one can now evaluate the interfacial tension from Eq (8). 
Now let us turn to three phase coexistence which is what we encounter in the ternary mixture. To calculate the interfacial properties within the Landau theory, we simply write down a function $f(M)$ with three minima, at $M_{0}, 0,-M_{0}$ say, and solve for the profile as before. We need not carry this out to obtain an important result concerning the oil water interface, a result which is most easily seen from the classical mechanics analogy. Our terrain now consists of three hills. Our particle starts on the leftmost hill and is to end up on the rightmost hill. It is clear, from conservation of energy, that before the particle gets there, it will come to rest (spend an infinite time) at the center hill. Translated to the statistical mechanics problem, this means that there will be an infinite thickness of the middle phase sitting between the oil and water phases, i.e. the middle phase will always wet the oil water interface within this Landau theory. Yet this result is contrary to experiment.

What is wrong with this approach? In the case of amphiphilic systems, the problem is that the simple Landau theory does not permit a correct description of the amphiphile. Because of the nature of the amphiphile, it likes oil to be near water. It therefore favors gradients in the order parameter. This can be put into the Landau theory by letting the coefficient of the gradient squared term in Eq. (1) be negative. However, if it is negative, the functional of Eq. (1) has no minimum. This can be easily remedied (4) by adding a term proportional to the square of the Laplacian of $M$, that is we replace the functional of Eq. (1) by

$$
F\{M(\mathbf{r})\}=\int d^{3} r\left[c\left(\nabla^{2} M\right)^{2}+g(M)(\nabla M)^{2}+f(M)\right],
$$

where $c>0$. Note that the coefficient $g$ generally depends on $M$ because we expect $g$ to be negative for values of $M$ which describe the middle phase, but to be positive for $M$ in the oil or water phases. One can see that this Landau form is correct for amphiphilic systems because the structure function which one obtains from it is (c.f. Eq. 4)

$$
<M_{\mathbf{q}} M_{-\mathbf{q}}>\propto \frac{1}{c q^{4}+g q^{2}+\omega},
$$

which, for $g<0$ has a peak at a non-zero value of $\mathrm{q}$, the signature of a structured fluid. For the correlation function $G(\mathbf{r})$ one finds that it decays exponentially with the same correlation length $\xi \equiv \sqrt{g / \omega}$ as before provided that $\gamma \equiv g / \sqrt{4 \omega c}>1$, while for $1>\gamma>-1$

$$
G(\mathbf{r})=\frac{\pi \lambda \tilde{\xi}}{2 c r} \exp (-r / \tilde{\xi}) \sin (2 \pi r / \lambda)
$$

There are now two lengths (5), the correlation length $\tilde{\xi}$

$$
\tilde{\xi}^{2}=\xi^{2}\left[\frac{1}{\gamma(1+\gamma)}\right]
$$

and the wavelength $\lambda$

$$
\left(\frac{\lambda}{2 \pi}\right)^{2}=\xi^{2}\left[\frac{1}{\gamma(1-\gamma)}\right]
$$

The oscillatory behavior of $\mathrm{Eq}(16)$ reflects the ordering properties of the amphiphile. Note that this oscillatory behavior vanishes at the disorder line (6) given by $\gamma=1$.

We can now return to the problem of three coexisting phases to see whether we have, in fact, fixed up the problem as we intended. We must now extremize (c.f. Eq 8)

$$
\sigma\{M\} \equiv \int d z\left[c\left(\frac{d^{2} M}{d z^{2}}\right)^{2}+g(M)\left(\frac{d M}{d z}\right)^{2}+f(M)\right]
$$


In the classical mechanics analogy, this would lead to a strange dynamics

$$
-2 c \frac{d^{4} x}{d t^{4}}+\frac{1}{2} \frac{d m}{d x}\left(\frac{d x}{d t}\right)^{2}+m \frac{d^{2} x}{d t^{2}}=-\frac{d V}{d x},
$$

with the constant of motion

$$
2 c\left[\frac{1}{2}\left(\frac{d^{2} x}{d t^{2}}\right)^{2}-\frac{d x}{d t} \frac{d^{3} x}{d t^{3}}\right]+\frac{m(x)}{2}\left(\frac{d x}{d t}\right)^{2}+V(x)=E .
$$

Clearly our intuition from classical mechanics is no longer of any use. But we can see that even when the energy $E=0$, the particle can pass through the top of the middle hill where $V(x)=0$ with a non-zero velocity because the sign of the new first term can be opposite that of the "kinetic energy" term. The result for the statistical mechanics problem is that the middle phase need not wet the water oil interface.

The Euler Lagrange equation, $\mathrm{Eq}_{\mathrm{q}}(20)$ or its statistical mechanics analogue, is not readily soluble with an arbitrary free energy per unit volume $f(M)$ and arbitrary coefficient $g(M)$. However, when $f(M)$ is taken to be a piecewise continuous and parabolic function, and $g(M)$ is taken to be piecewise constant, such as

$$
\begin{aligned}
f(M) & =\omega\left(M+M_{0}\right)^{2} / 2 \quad M<-M_{0} / 2, \\
& =\omega M^{2} / 2 \quad-M_{0} / 2<M<M_{0} / 2, \\
& =\omega\left(M-M_{0}\right)^{2} / 2 \quad M_{0} / 2<M,
\end{aligned}
$$

and

$$
\begin{array}{rlrl}
g(M) & =g_{1}>0, & M<-M_{0} / 2, \\
& =g_{2}, & & -M_{0} / 2<M<M_{0} / 2, \\
& =g_{1}, & & M_{0} / 2<M,
\end{array}
$$

then the Euler Lagrange equation becomes a linear ordinary differential equation with constant coefficients and the problem is easily solved. The profile is a sum of exponentials, and if all $\gamma_{i} \equiv g_{i} / \sqrt{4 \omega c}>1$, then all exponents are real, while if some $-1<\gamma_{i}<1$, then some exponents are complex. It is in the middle phase that we expect this to happen. Once the profile is calculated, the interfacial tension can be obtained as before. The only tension which gives any difficulty is the oil water tension. For this, we allow a thickness $L$ of middle phase to come between the oil and water phases, calculate $\sigma(L)$, and then minimize this tension with respect to the thickness. This minimum gives the actual tension, and the value of $L$ for which it occurs gives the actual thickness. If $L$ is infinite, the middle phase wets the oil water interface, whereas if it is finite, it doesn't. From the three interfacial tensions, the contact angle of a pendant drop can be obtained ( 7 ).

For the case of the balanced system, one finds (4) that when $\gamma_{2}>1$, the interface potential decays at large $L$ monotonically and exponentially with $L$, just as the bulk correlation function of Eq. (5) does. Consequently, the minimum of $\sigma(L)$ occurs at an infinite value of $L$ and the middle phase wets the oil water interface. However, when $\left|\gamma_{2}\right|<1, \sigma(L)$ at large $L$ is a damped oscillatory function, just as is the bulk correlation function of $\mathrm{Eq}$. (16). Consequently, its minimum occurs at a finite value of $L$ so that the middle phase does not wet the oil water interface. We see, therefore, that the failure of the middle phase to wet the oil water interface is a direct consequence of the fact that it is a structured fluid. For short range forces, we predict a continuous transition at the disorder line itself (at which $\gamma_{2}=1$ ). When long range forces are included (4), one finds that the transition occurs on the structured side of the disorder line and 
is first order. That the wetting transition does occur on the structured side of the disorder line has recently been verified (1).

We have now understood the first set of experimental measurements referred to in the introduction. In order to understand the second, we apply the same theory to the case in which a critical endpoint is approached (8). The equilibrium values of the order parameter in the three coexisting phases are taken to be $M_{1}, M_{2}=0$, and $M_{3}=-m_{0} M_{1}$. The critical endpoint occurs as $m_{0} \rightarrow 0$, and phases 2 and 3 become critical. We approximate $f(M)$ by the piecewise continuous function

$$
\begin{aligned}
f(M) & =\omega M_{1}^{2}\left(\frac{M}{M_{1}}-1\right)^{2} \quad M_{12} \leq M \\
& =\omega M_{1}^{2} m_{0}^{2}\left(\frac{M}{M_{1}}\right)^{2} \quad M_{23} \leq M \leq M_{12} \\
& =\omega M_{1}^{2} m_{0}^{2}\left(\frac{M}{M_{1}}+m_{0}\right)^{2} \quad M \leq M_{23}
\end{aligned}
$$

where $M_{12}=M_{1} /\left(1+m_{0}\right)$, and $M_{23}=-m_{0} M_{1} / 2$. Note from Eq. 24 that the curvature of the parabolas describing phases 2 and 3 decrease in proportion to the square of the order parameter difference $M_{2}-M_{3}=m_{0} M_{1}$ as the endpoint is approached, just as in mean field theory. For $g(M)$ we take the piecewise constant form

$$
\begin{aligned}
g(M) & =g_{1}, & & M_{12}<M, \\
& =g_{2}, & & M_{23}<M<M_{12}, \\
& =g_{3}, & & M<M_{23} .
\end{aligned}
$$

Several choices remain as to the parameters which describe the three phases. The spectator phase is essentially described by the single parameter $u \equiv g_{1} / \sqrt{4 \omega c}$. This phase will be an ordinary fluid, as opposed to a microemulsion, provided that $u>1$. The properties of phases 2 and 3 are set by the ratios $g_{2} / g_{1}$ and $g_{3} / g_{1}$, which determine the relative difficulty of bringing about variations in the order parameter $M(z)$ in these phases. Physically, this should depend on the concentrations of amphiphile in them, concentrations which vary as the critical endpoint is approached. Thus we let these ratios depend upon $m_{0}$. We choose the linear dependence

$$
\begin{aligned}
& g_{2}\left(m_{0}\right)=g_{1}\left[r-m_{0}\left(r-\frac{s}{u}\right)\right], \\
& g_{3}\left(m_{0}\right)=g_{1}\left[r+m_{0}(1-r)\right] .
\end{aligned}
$$

Because the concentration of amphiphile in phase 3 increases as the critical endpoint is approached, we expect it to become less difficult to bring about spatial variations in the order parameter. Hence we have chosen $g_{3}$ to decrease along this path, i.e. as $m_{0} \rightarrow 0$. Similarly, the concentration of amphiphile in phase 2 decreases, so that we have chosen $g_{2}$ to increase along this path. When $m_{0}=1$ and the order parameters in phases 1 and 3 are equal and opposite, we have required the system to be "balanced" by imposing the condition $g_{3}(1)=g_{1}$. In a balanced system, the middle phase has its largest concentration of amphiphile, and is therefore closest to being unstable to a lamellar phase. In order to ensure that it is stable, our parameterization requires $-1<s$. The closer $\mathrm{s}$ is to -1 , the more structured is the microemulsion. Near the critical endpoint, $g_{2}$ and $g_{3}$ approach the common value $r g_{1}$. Hence, the smaller the value of $r$, the less resistant are these phases to spatial variations in the order parameter. When $r=0$, the endpoint is a Lifshitz critical point (9). 
From these considerations, we model poor amphiphiles by a larger value of $r$ and smaller value of $|s|$, and good amphiphiles by a smaller $r$ and larger $|s|$. Figures 2 and 3 show results for a series of seven increasingly strong amphiphiles. The contact angle is shown as a function of $m_{0}^{2}$ because, in mean field theory, this is proportional to the temperature when close to the critical endpoint. Hence the figures are more directly comparable to Fig. 1. The system is balanced at $m_{0}^{2}=1$, and the endpoint occurs at $m_{0}^{2}=0$. The value of $u=1.58$ in all cases.

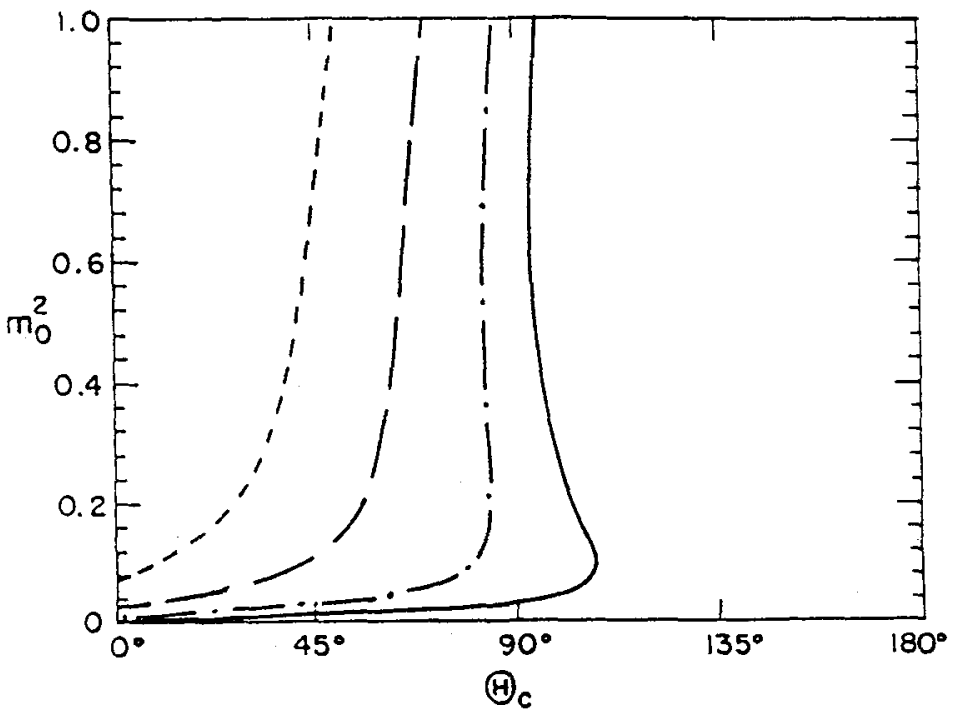

Fig. 2 Calculated behavior of the contact angle vs. the square of the reduced order parameter $m_{0}^{2}$. The critical endpoint is at $m_{0}^{2}=0$. In order of increasing amphiphilic strength, the curves are shown short dashed, long-dashed, dot-dashed, and solid.

In Fig. 2, the ratios $(r, s)$ are $(0.2,0$.$) , dotted line; (0.15,-0.237)$, dashed line; $(0.1,-0.395)$, dot-dashed line: $(0.075,-0.474)$, solid line. The qualitative agreement with either the upper or lower half of Fig. 1 is clearly good. In Fig. 3, the amphiphiles are stronger than those of Fig. 2. They are characterized by $(r, s)$ of $(0.05,-0.474)$, dashed line; $(0.04,-0.505)$, dot-dashed line; $(0.03,-0.617)$ solid line. In the last two cases, the contact angle actually reaches $180^{\circ}$ where a wetting transition occurs in which phase 3 wets the 1-2 interface. As the endpoint is approached. a dewetting transition takes place and, even closer to the endpoint, another wetting transition at which 2 wets the $1-3$ interface.

A wetting transition occurs in all cases before the critical endpoint is reached, in accord with the Cahn argument. In fact the behavior of the contact angle can be explained in the light of this argument. Let us begin with the balanced system for which no phase wets the interface between the other two. Although the Cahn argument predicts a wetting transition, it does not state whether the contact angle of the pendant drop we have been discussing should decrease, indicating that phase 2 will wet the 1-3 interface, or should increase, indicating that phase 3 will wet the 1-2 interface. Which of these should occur depends upon the sign of the field which couples to the difference $M_{2}-M_{3}$. Normally, this field arises simply from the fact that variations in the order parameter $M(z)$ are costly. Thus the spectator phase, 1 , prefers the middle phase 2 over phase 3 , because the value of the order parameter in phase $1, M_{1}$, is closer to the value in phase $2, M_{2}$, than it is to the value in phase 3 . This preference is the greater in proportion to the difference $M_{2}-M_{3}$, and thus acts like a field coupling to the order parameter. Let the sign of this field be positive in this case. By itself, this field favors the decrease of the contact 
angle, and the wetting of the 1-3 interface by phase 2. The analysis is quite different when the interface with phase 1 is coated with amphiphile, because the amphiphile prefers to have large gradients in the order parameter. Therefore, it prefers phase 3 to sit next to phase 1 , rather than a macroscopic amount of phase 2. The effect of the amphiphile, then, is to produce a field which is negative, of opposite sign to that produced by phase 1 alone. By itself, this field favors the increase of the contact angle, and the wetting of the 1-2 interface by phase 3 . The actual behavior of the contact angle depends on the competition of these two tendencies as reflected in the net sign of the effective field. In a balanced system with a good amphiphile, the effect of the amphiphile is dominant and the contact angle initially increases. However as one moves toward the endpoint, the amount of amphiphile in the middle phase decreases, and it becomes less structured. The influence of the amphiphile weakens, so that the effective field changes sign, and the contact angle decreases. This field ultimately causes a wetting transition as predicted by the Cahn argument.

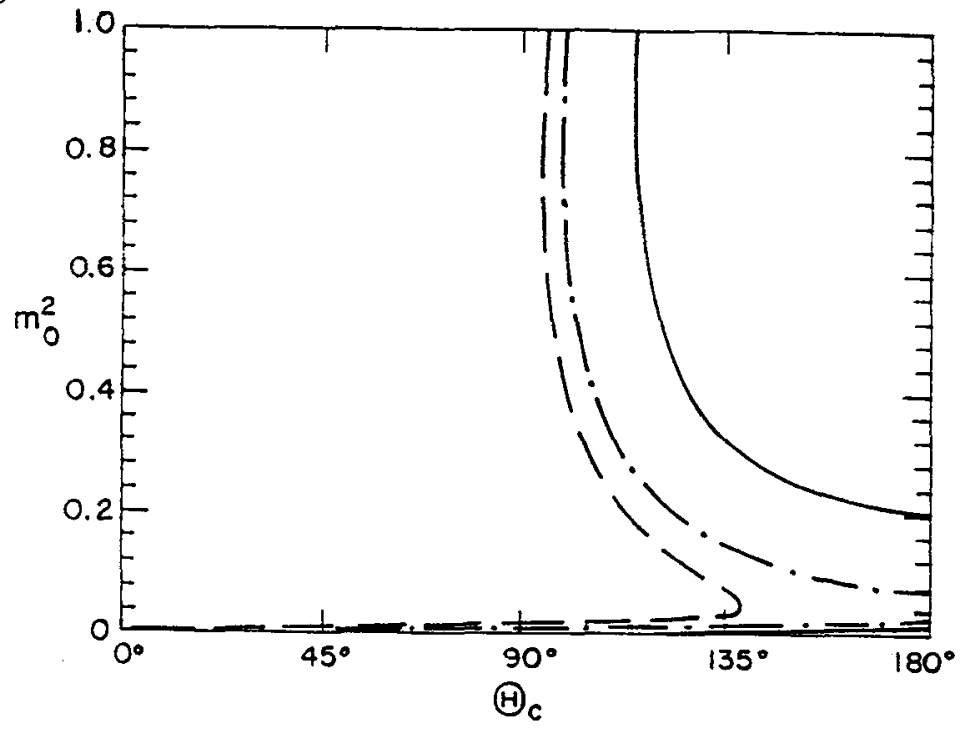

Fig. 3 Same as in Fig. 2, but the amphiphiles are stronger. In order of increasing amphiphilic strength, the curves are shown dashed, dot-dashed, and solid.

To summarize, we have seen that the wetting properties of microemulsions are directly related to the fact that the microemulsion is a complex fluid, one which displays a tendency to order on a length scale unrelated to the bulk correlation length. By extending the usual Landau treatment of interfaces to encompass this tendency, we can employ this procedure to predict the interfacial properties of these systems. The predictions that the wetting transition in a balanced system will be first order and occur on the structured side of the disordered line have already been confirmed. The prediction that, in a system with a very good amphiphile, the contact angle of the middle phase can actually reach $180^{\circ}$, producing the analogue of a "drying" transition, has yet to be tested.

Finally, I wish to address the origin of the ultralow oil water tensions when the oil- and water-rich phases coexist with a microemulsion made with a good amphiphile. It is often stated that this low tension is associated with a bulk critical point, but this is not so. If it were, one would expect the oil-rich and water-rich phases to be relatively close in composition to one another. In actuality, the composition of these phases is that of relatively pure oil or water. If a critical point is not the origin of this low tension, what is? 
To answer this question, consider a balanced system in which we have a lamellar phase, and let us imagine that, by decreasing the amphiphile concentration, we could increase the spacing between the lamellae without limit until we were left with a single amphiphile sheet separating the bulk oil-rich phase from the water-rich phase. (This scenario is often referred to as a continuous unbinding transition.) Just at the point at which the spacing diverges, we would have three phase coexistence between oil-rich, water-rich, and lamellar phases. It would be a particularly interesting three-phase coexistence however. Because the wavelength characterizing the lamellar phase is infinite, the amphiphile concentration in all three phases would be identical; the usual three phase triangle would have collapsed to a three phase line. It is easy to see that, under these conditions, the oil water interfacial tension, $\sigma_{O W}$ is identically zero: the free energies of all three phases must be identical, and the free energy of the lamellar phase differs from those of the other two only by an amount $\sigma_{O W}-B$ per unit wavelength, where $B$ is the binding energy of the lamellae. This difference must be zero at three phase coexistence, hence the interfacial tension is equal to the binding energy. This is true even when the lamellar phase is characterized by a finite wavelength, but when, as we are envisioning, this wavelength diverges, the binding energy vanishes. Thus the oil water tension vanishes also. We have produced, therefore, a situation in which the oil and water phases coexist with a middle phase and with one another, yet the tension between oil and water phases vanishes (10). Furthermore, the amphiphile is an extraordinary solubilizer in that no additional amount of amphiphile is needed to solubilize the oil and water in the middle phase than already exist in the oil- and water-rich phases. Finally, as I have not used the symmetry of the lamellar phase in this argument, I can let the middle phase be a disordered one, in which case we have produced the ideal microemulsion.

I cannot resist the following argument to indicate that the ideal microemulsion does not wet the oil water interface. Assume that it does not. Then the picture of the middle phase as coherent regions of bulk oil and of bulk water separated by microscopically thin regions of amphiphile is a self consistent one. If, on the other hand, we assume that the middle phase wets the oil water interface, then any two adjoining colnerent regions of oil and water in the middle phase cannot be adjoining at all, but must be separated by a macroscopically thick region of more middle phase, and so on ad infinitum. As this picture is not self-consistent, I conclude that the ideal microemulsion does not wet the oil water interface.

I also want to note that this picture of the ideal microemulsion, which differs from the other two phases with which it coexists only in the difference in oil and water concentrations, and not in the amphiphile concentration, lends further support to our earlier Landau treatment in which we used only the difference in oil and water concentrations to label the three phases, rather than employing a Landau expansion in two independent concentrations.

A real microemulsion differs from the ideal one, of course. The amphiphilic sheets cannot be separated an infinite distance before three-phase coexistence is encountered. The maximum distance they can be pulled apart before coexistence is reached is thought to be on the order of the persistence length (11). When the sheets are rather rigid, and this distance is large, the height of the three phase triangle will not be zero, but it will be smail, so that the amphiphile is still a very good solubilizer. Also, as the sheets are separated by a finite distance, their binding energy will be non-zero. But if their spacing is large, this binding will be small, and therefore the water oil tension will be also. These arguments will apply whether the middle phase is lamellar, or a disordered microemulsion.

We see, then, how the interfacial properties of amphiphilic systems follows from the self assembling nature of the amphiphiles which bring about a structured fluid. It was shown by explicit calculation that this structure was directly reflected in the wetting properties of these systems. Finally, we saw how the scale of this structure is related to the low interfacial tensions between coexisting oil and water phases.

I am grateful to Prof. M. Kahlweit for sending me a preprint of Ref. 1. I also thank J. 
Cahn, M. Kahlweit, S. Milner, R. Strey, and T. Witten for stimulating conversations, and am indebted to G. Gompper, J. Putz, and R. Hotyst for fruitful collaborations. This work was supported in part by the National Science Foundation under Grant No. DMR-8916052.

\section{References}

1. K.-V. Schubert and R. Strey, J. Chem. Phys. 95, 8532 (1991).

2. J.W. Cahn, J. Chem. Phys. 66, 3667 (1977).

3. M. Aratono and M. Kahlweit, J. Chem. Phys. 95, 8578 (1991).

4. G. Gompper and M. Schick, Phys. Rev. Lett. 65, 1116 (1990).

5. M. Teubner and R. Strey, J. Chem. Phys. 87, 3195 (1987).

6. M.E. Fisher and B. Widom, J. Chem. Phys. 50, 3756 (1969); J. Stephenson, J. Math. Phys. 11,420 (1970).

7. J.S. Rowlinson and B. Widom, Molecular Theory of Capillarity (Clarendon, Oxford, 1982), p211.

8. J. Putz, R. Hołyst, and M. Schick, Phys. Rev. A (in press).

9. G. Gompper, R. Hotyst, and M. Schick, Phys. Rev. A43, 3157 (1991).

10. M. Schick and W.-H. Shih, Phys. Rev. B34, 1797 (1986).

11. L. Golubovic and T.C. Lubensky, Phys. Rev. A41, 4343 (1990). 